Meta

Journal des traducteurs

Translators' Journal

\title{
Masculin - féminin
}

\section{Irène V. Spilka}

Volume 20, numéro 4, décembre 1975

URI : https://id.erudit.org/iderudit/004245ar

DOI : https://doi.org/10.7202/004245ar

Aller au sommaire du numéro

Éditeur(s)

Les Presses de l'Université de Montréal

ISSN

0026-0452 (imprimé)

1492-1421 (numérique)

Découvrir la revue

Citer cet article

Spilka, I. V. (1975). Masculin - féminin. Meta, 20(4), 263-270.

https://doi.org/10.7202/004245ar

Ce document est protégé par la loi sur le droit d'auteur. L'utilisation des services d'Érudit (y compris la reproduction) est assujettie à sa politique d'utilisation que vous pouvez consulter en ligne.

https://apropos.erudit.org/fr/usagers/politique-dutilisation/
Cet article est diffusé et préservé par Érudit.

Érudit est un consortium interuniversitaire sans but lucratif composé de l’Université de Montréal, l'Université Laval et l'Université du Québec à Montréal. Il a pour mission la promotion et la valorisation de la recherche. https://www.erudit.org/fr/ 


\section{Masculin - féminin *}

0 . Nos façons de parler et d'écrire reflètent-elles nos préjugés même inconscients, notamment le préjugé «sexiste » qui consiste à croire qu'une partie de l'humanité est supérieure à l'autre et que féminin signifie deuxième sexe ? Sans vouloir faire de la psychanalyse on peut affirmer que la coutume d'interdire aux femmes - et aux hommes - l'accès à certains emplois est dépourvue de fondement rationnel et repose sur des notions périmées concernant à la fois les caractères sexuels secondaires et la nature des occupations professionnelles. La question intéresse tout particulièrement les rédacteurs d'appels de services et les traducteurs. En effet, par le choix des termes dont ils se composent, ces textes peuvent marquer une préférence pour un sexe ou l'autre; leur large diffusion par la presse et autres moyens de communication contribue à maintenir au sein du public l'impression que seul les hommes - ou les femmes - sont aptes à exercer tel métier ou telle profession qui, en réalité, n'exige aucune disposition particulièrement féminine ou masculine. S'il ne semble pas absolument nécessaire de forcer les femmes à devenir mineurs et les hommes à se faire bonnes d'enfant, il est cependant souhaitable de laisser à chacun la liberté de choisir son travail et son style de vie en fonction de ses goûts et de ses talents naturels. Récemment, la maison McGraw-Hill publiait des conseils aux rédacteurs, les encourageant à substituer aux appellations traditionnelles telles que fireman, qui désignent obligatoirement des hommes, des termes englobant les deux sexes, comme firefighter, quitte à les inventer au besoin.

On sait que le français actuel est moins dynamique que l'américain contemporain et hésite à rompre avec la tradition pour créer des néologismes dont la nécessité ne se fait pas sentir de façon impérieuse. Par ailleurs, la structure du français est différente de celle de l'anglais et comporte des contraintes particulières concernant la catégorie du genre. Il convient donc d'examiner les rapports du genre grammatical au genre naturel pour savoir s'il est possible de neutraliser le contenu des annonces d'emplois de manière à ce qu'elles touchent également les deux sexes. C'est le résultat d'un premier tour d'horizon que nous livrons aujourd'hui aux lecteurs de Meta.

\footnotetext{
* Le présent article est tiré d'un rapport rédigé à la demande du Ministère des Affaires des anciens combattants.
} 
1. Grammaire et sémantique. - En français, le genre est une catégorie du nom, ce qui signifie que tous les noms ont un genre, le masculin ou le féminin. Le plus souvent, celui-ci est déterminé par la forme du nom, c'est-à-dire la suite de sons qui composent ce dernier. Ainsi, les mots terminés par -ation sont du féminin et tous les mots terminés par-acre sont du masculin, sauf nacre, qui est du féminin (l'usage canadien ignore souvent cette exception à la règle). Il existe des règles pour rendre compte des rapports du genre à la forme, mais il ne semble pas utile de les présenter ici, tous les dictionnaires faisant suivre chaque nom de l'indication $n . m$. ou n.f. (nom masculin, nom féminin).

Le genre d'un nom est attesté par le jeu des pronoms de substitution. Tout nom masculin, sans exception, se remplace par il et tout nom féminin par elle : le mousse s'appelle Jean, IL a quinze ans, mais la mousse au chocolat est bonne, ELLE est exquise. Le français n'a pas de neutre, en ce sens que les noms sont toujours remplaçables par il ou par elle, mais certaines formes des morphèmes grammaticaux sont cependant indifférenciés. Tels sont :

- le pronom clitique pluriel de l'accusatif les : mes seurs, épousez-LES;

- les clitiques du datif y, lui, leur : regarde maman, dis-LUI bonjour ;

- le clitique de l'ablatif en: la carafe, j'EN verse le contenu;

- les pronoms démonstratifs indéfinis ce, cela, ça: la glace à la vanille, ÇA, c'est bon ;

- les pronoms possessifs collectifs pluriels les nôtres, les vôtres, les leurs: nos cheveux sont noirs, LES VOTRRES sont blonds; nos robes sont longues, LES VOTRES sont courtes;

- les formes plurielles des déterminants du nom les, des, ces: LES gargons et LES filles ;

- l'article élidé l' : L'ami et L'amie ;

- les adjectifs possessifs du singulier collectif notre, votre, leur : NOTRE mère, NOTRE père ;

- les formes plurielles des adjectifs possessifs mes, tes, ses, nos, vos, leurs: LEURS oncles et LEURS tantes;

- les formes mon, ton, son lorsqu'elles précèdent un nom ou un adjectif commençant par une voyelle ou un $h$ muet: MON amie, TON horrible cousine.

$\mathrm{Si}$ nous énumérons longuement des faits parfaitement connus du locuteur francophone, c'est pour faire sentir à quel point le genre grammatical français peut être ambigu. C'est en effet bien plus une propriété grammaticale du substantif qu'une catégorie sémantique du nom. Du moins, conviendrait-il de distinguer soigneusement les deux et de les marquer par des signes distincts, par exemple $f$ et m pour désigner la catégorie grammaticale, la chaise (f), mais FEM et MASC pour indiquer le trait sémantique, la belle-mère (FEM). Les substantifs de l'un OU de l'autre genre grammatical pouvant s'appliquer à des êtres de l'un ET de l'autre sexe porteraient alors les indications convenables : la recrue (f, MASC-FÉM), le peintre ( $\mathrm{m}, \mathrm{MASC}-\mathrm{FÉM}$ ), la mouche (f, MASC-FÉM), le moustique (m, 
MASC-FÉM). La catégorie $\mathrm{f}$ et $\mathrm{m}$ ainsi que la dimension FEM et MASC étant binaires, il est évidemment redondant de marquer chaque pôle de l'opposition par un signe particulier, et l'on peut se contenter de faire précéder un symbole, choisi arbitrairement, des signes + et - selon le cas : la recrue $(-\mathrm{m}, \pm \mathrm{MASC})$, le peintre $(+\mathrm{m}, \pm$ MASC), ce qui satisfait le principe de l'économie des signes.

2. Économie linguistique. - Le masculin occupe la place prépondérante en français. Le nombre des substantifs masculins est, en effet, supérieur à celui des substantifs féminins. L'attraction du masculin se manifeste dans certaines classes de néologismes, où l'on aurait pu s'attendre à voir surgir des féminins. Sur le modèle des noms d'appareils comme balayeuse, essoreuse, fraiseuse, valseuse, berline, carabine, limousine, turbine, bicyclette, motocyclette, braisière, daubière, glacière, sorbetière, calculatrice et tant d'autres, il eut été facile de créer " ordinatrice », mais c'est ordinateur qui l'a emporté, comme dans un autre domaine, formant et composant l'emportent sur formante et composante dans maints textes techniques.

En outre, les règles de concordance : accord des adjectifs et des participes, et substitution des pronoms favorisent toujours le masculin lorsque deux ou plusieurs noms de genres différents en sont affectés. Toutes ces règles, généralement citées séparément dans les grammaires traditionnelles, peuvent se résumer dans une seule règle de composition, que l'on schématise ainsi :

\begin{tabular}{c|c|c}
$*$ & $\mathrm{M}$ & $\mathrm{F}$ \\
\hline $\mathrm{M}$ & $\mathrm{M}$ & $\mathrm{M}$ \\
\hline $\mathrm{F}$ & $\mathrm{M}$ & $\mathrm{F}$
\end{tabular}

Exemples : Pierre et Marie sont beaux.

Jean et Jeanne sont-ils là?

Une revue et un livre anglais.

On notera cependant que la copule être peut relier deux noms de genres différents :

Exemples: Son mari est une brute.

Sa femme est un génie.

3. Nominaux, indéfinis et collectifs. - Le français possède des mots permettant de parler des personnes d'une façon générale et indéfinie. Employés sans précision du sexe ni référence à un être particulier, les indéfinis comme chacun, nul, personne (négatif), quelqu'un s'emploient au masculin, même lorsqu'on s'adresse à un auditoire féminin : personne n'est venu; (en parlant à des femmes) quelqu'un veut-il proposer l'adoption de cette proposition? Il faut cependant noter que personne employé comme nom et à l'affirmatif est toujours féminin et désigne aussi bien un homme qu'une femme: il n'y a plus qu'une personne, c'est un monsieur : toute personne désireuse de se présenter au concours (homme ou femme). Enfin, quand un et ses composés sont déterminés dans la phrase, ils suivent les règles d'accord : aucune d'entre elles. 
Le nom collectif gens, toujours pluriel, suit des règles d'accord particulières. Comme il sert à désigner certaines catégories professionnelles, comme gens de maison (domestic help), il n'est pas inutile de les rappeler. Lorsqu'un adjectif dont la forme varie selon le genre précède gens, il se met au féminin : de bonnes gens; mais après gens, il se met au masculin : des gens intelligents. Par souci d'esthétique, on évite les tournures comme de bonnes gens intelligents.

4. Un cas d'homonymie. - Malgré ce qui a été dit au paragraphe 1, le genre n'est pas toujours lié à la forme et certaines formes se retrouvent sous les deux genres. La liste suivante est incomplète mais suffisante pour illustrer ce cas particulier d'homonymie :

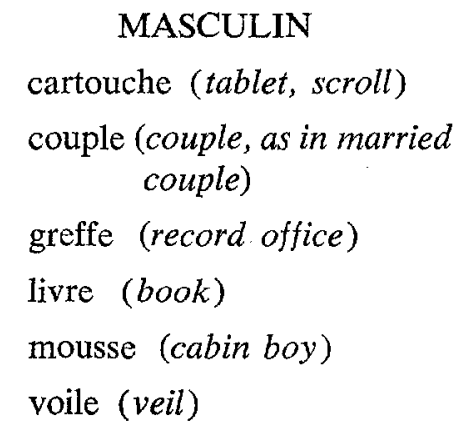

FÉMININ
cartouche (cartridge)
couple (two)
greffe (graft, scion)
livre (pound)
mousse (moss, lather)
voile (sail)

Si nous le relevons ici, c'est qu'il intéresse directement le problème des désignations de fonction, d'occupation et d'emploi. Un mousse est bien un garçon, mais une mousse ne saurait être une jeune fille puisque le féminin sert déjà à désigner divers objets, à moins que l'on veuille introduire un néologisme sémantique hardi, au reste immotivé, et se servir du même mot pour une personne et une chose, comme dans le cas de cuisinière.

5. Les formes invariables. - Le genre grammatical ne constitue pas nécessairement une indication du genre naturel. De nombreux noms d'animaux ne connaissent qu'une forme pour le mâle et la femelle : girafe, tortue, et mouche sont toujours féminins (,$- \pm \mathrm{MASC}$ ), rhinocéros, serpent et moustique sont toujours masculins $(+\mathrm{m}, \pm$ MASC). On précise, au besoin, le sexe de l'animal à l'aide des épithètes mâle et femelle. De même, certains noms de personnes ne connaissent qu'une forme, masculine ou féminine: une vigie et une sentinelle sont presque toujours des hommes, un laideron et un bas-bleu ne se disent qu'en parlant d'une femme. En termes de jurisprudence, un ascendant et un conjoint désignent l'homme ou la femme; si l'on désire préciser le sexe, il faut recourir à un synonyme, comme père, mère, mari, épouse, etc.

6. Les noms épicènes. - Certains noms ont la propriété d'appartenir aux deux genres sous une même forme : selon le sexe de la personne, on dira un ou une enfant, un ou une soprano, un ou une Russe. La liste de ces noms, souvent terminés par un $e$, est donnée par les grammaires. Les dictionnaires pourraient ajouter la mention ( $\pm \mathrm{m}, \pm$ MASC $)$. 
7. Les formes variables $d u$ masculin et du féminin. - Un très grand nombre de noms de personne sont marqués quant au genre :

$\begin{array}{ll}\text { MASCULIN } & \text { FÉMININ } \\ \text { colonel } & \text { colonelle } \\ \text { gardien } & \text { gardienne } \\ \text { cadet } & \text { cadette } \\ \text { préfet } & \text { préfète } \\ \text { fermier } & \text { fermière } \\ \text { danseur } & \text { danseuse } \\ \text { inspecteur } & \text { inspectrice } \\ \text { maître } & \text { maîtresse } \\ \text { sacristain } & \text { sacristine }\end{array}$

Les règles de formation du féminin, que l'on trouve dans les grammaires, sont connues de toute personne francophone instruite.

L'intérêt de cette classe réside dans le fait que le genre grammatical correspond, cette fois, à une distinction sémantique, au cotnraire de ce qui se passe pour les noms ayant une forme unique, signalés au paragraphe 5. Un gardien désigne un homme qui fait fonction de gardien, et une gardienne, la femme qui remplit les mêmes fonctions, contrairement à une ordonnance, une estafette, un dentiste et un magistrat, qui ne fournissent aucune indication sur le sexe de la personne désignée.

Deux substantifs de la même famille peuvent présenter une forme masculine et une forme féminine sans que celles-ci dénotent pour autant le trait sémantique correspondant : médecin $(+\mathrm{m}, \pm \mathrm{MASC})$ n'est pas l'homologue de médecine ( - m, - ANIMÉ).

8. Valeur distinctive de quelques féminins. - Certains noms féminins ont un sens spécialisé par rapport à une autre forme féminine, ou au masculin. Tels sont :

\begin{tabular}{|c|c|}
\hline $\begin{array}{l}\text { MASCULIN } \\
\text { avocat }\end{array}$ & $\begin{array}{l}\text { FÉMININ } \\
\text { (femme) avocat }=\text { inscrite au barreau } \\
\text { avocate = intermédiaire }\end{array}$ \\
\hline facteur & $\begin{array}{l}\text { factrice = qui passe les lettres } \\
\text { facteur = (femme) agent, mandataire, etc. }\end{array}$ \\
\hline directeur & $\begin{array}{l}\text { directrice = d'une école primaire, secondaire } \\
\text { directeur = d'un bureau, d'un service, etc. }\end{array}$ \\
\hline agent & agente $=$ terme péjoratif \\
\hline & doctoresse $=$ terme péjoratif \\
\hline poète & poétesse $=$ terme péjoratif \\
\hline
\end{tabular}

9. Genre précisé à dessein. - Lorsqu'il n'existe pas d'appellation féminine pour désigner un emploi, une fonction ou une dignité traditionnellement réservés aux hommes, il est toujours possible de préciser le féminin en ajoutant femme, madame, etc. : une femme médecin, Madame le Maire. 
10. Genre grammatical lié au sexe. - Certaines occupations ne peuvent, en raison de leur caractère sexuel, intéresser que les hommes ou les femmes, mais pas les deux. Nourrice et castrat appartiennent à cette catégorie. De semblables distinctions, fondées sur le genre naturel, se retrouvent ailleurs dans la langue. Ainsi, la terminologie des liens de parenté comporte deux classes de noms reflétant à la fois le genre et la relation de parenté.

11. Rapports du genre grammatical au genre naturel. - Il est facile de conclure, d'après ce qui précède, que l'expression du genre naturel par le genre grammatical, en français, oscille entre l'ambiguïté totale et la précision inéluctable. Le tableau récapitulatif qui suit met en lumière les rapports du sens et de la forme dans la catégorie du genre.

TABLEAU RÉCAPITULATIF

\begin{tabular}{|c|c|c|}
\hline Rapport & Marque & Exemples \\
\hline \multirow{2}{*}{ Nul } & $\begin{array}{l}\text { Genre grammatical } \\
\text { fonction de la } \\
\text { syntaxe }\end{array}$ & $\begin{array}{l}\text { quelqu'un (indéf. } m \text { ) } \\
\text { personne (nég. } m \text { ) } \\
\text { une personne (affirm. } f \text { ) } \\
\text { des gens (coll. } m / f \text { ) }\end{array}$ \\
\hline & $\begin{array}{l}\text { Genre grammatical } \\
\text { inhérent au nom }\end{array}$ & $\begin{array}{l}\text { une recrue (homme ou femme) } \\
\text { un critique " }\end{array}$ \\
\hline $\begin{array}{l}\text { Ambiguiité (en } \\
\text { l'absence du } \\
\text { déterminant) }\end{array}$ & $\begin{array}{l}\text { Nom invariable, } \\
\text { déterminant variable }\end{array}$ & $\begin{array}{l}\text { un / une concierge (homme } \\
\text { ou femme, tel qu'indiqué } \\
\text { par le déterminant) }\end{array}$ \\
\hline \multirow{2}{*}{ Correspondance } & $\begin{array}{l}\text { Nom et déterminant } \\
\text { variables }\end{array}$ & $\begin{array}{l}\text { un laborantin (homme) } \\
\text { une laborantine (femme) }\end{array}$ \\
\hline & $\begin{array}{l}\text { Formes variables avec } \\
\text { féminin spécialisé }\end{array}$ & $\begin{array}{l}\text { un gouverneur (homme) } \\
\text { une femme gouverneur } \\
\text { une gouvernante }\end{array}$ \\
\hline Obligatoire & $\begin{array}{l}\text { Formes uniques } \\
\text { marquées }\end{array}$ & $\begin{array}{l}\text { une nourrice } \\
\text { un donneur de sperme }\end{array}$ \\
\hline
\end{tabular}


12. La désignation des emplois et fonctions dans les appels de service. Lorsque le rapport du genre grammatical au genre naturel est nul, la rédaction d'appels de services égalitaires ne présente pas de difficulté. Si un journal cherche à engager un critique, homme ou femme, il doit forcément demander un critique, car une critique désignerait l'article qu'on souhaite faire écrire au critique. Il en va de même pour une ordonnance, une estafette, et une recrue qui n'ont pas de forme féminine correspondante. Lorsque le rapport est ambigu, en l'absence du déterminant, il suffit d'omettre ce dernier pour que l'appellation s'applique également aux hommes et aux femmes, mais on peut aussi utiliser le pluriel. En s'adressant à des aides, des arbitres, des artistes, des copistes, des libraires, des pianistes, des secrétaires, etc. on obtiendra éventuellement des candidats des deux sexes. Quand il existe deux formes différentes pour le masculin et le féminin, il faut prendre soin de les citer toutes les deux. On écrira donc boulanger ou boulangère, danseur ou danseuse, inspecteur ou inspectrice, ou encore acteur/actrice, maître/maîtresse, serviteur/servante. Par souci d'économie, les journaux ont parfois recours aux contractions du type un(e) employé(e), qui sont cependant impossibles avec des mots comme vendeur et vendeuse. Enfin, il est toujours permis d'expliciter : ingénieur (homme ou femme), ce qui ne laisse subsister aucun doute sur les intentions du rédacteur. S'il faut, au contraire, préciser que l'on ne s'adresse qu'à des femmes, on peut dire : une femme docteur, une femme exportateur.

13. L'accord anaphorique. - Lorsqu'on parle du docteur, Madame Une Telle, d'une femme peintre, etc., il peut sembler bizarre d'employer ensuite le pronom $i l$, sous prétexte qu'il remplace docteur, peintre. Il est parfaitement légitime, et de meilleur goût, d'accorder le pronom au genre de la personne. La même règle anaphorique s'applique aux autres éléments de l'énoncé. On dit et on écrit : ma collègue, le professeur N..., est très intelligente. Avec personne, cependant, l'accord se fait au féminin : la personne que nous recherchons sera âgée de quarante ans au moins; avec gens, il se fait au masculin : des gens de maison dévoués.

14. Questions de style. - Dans un texte suivi l'emploi des constructions du genre le/la représentant(e) ... il/elle sera diplômé(e) est gênant. Mieux vaut recourir au pluriel, les et des n'étant pas marqués quant au genre, et l'emploi de ils étant exigé par la règle (sans exception) de composition, l'ambiguité est suffisante pour qu'on ne sache s'il s'agit seulement d'hommes, ou d'hommes et de femmes : les représentants seront diplômés. Encore une fois, il est toujours possible de préciser qu'on s'adresse aux candidats masculins et féminins sans distinction. On trouvera au paragraphe 16 des exemples d'appels de services d'un caractère délibérément égalitaire.

15. Néologismes. - La création de postes nouveaux entraîne souvent la formation de désignations nouvelles. Il peut être opportun de préférer les terminaisons en -aire, -iste et $-o$, sur les modèles de fonctionnaire, copiste et sténodactylo, qui peuvent facilement devenir épicènes. Même si l'égalité des sexes au travail n'y trouve pas son compte, la tâche des futurs rédacteurs s'en trouvera allégée. 
16. A titre d'exemple:

- TÉLÉPHONISTES. Les intéressés se présenteront à nos bureaux munis de leur certificat d'études secondaires. La priorité sera accordée aux personnes bilingues. Belle apparence, bonne tenue exigées.

- CHEF D'EQUIPE - TRAITEMENT DES DONNÉES. La personne que nous recherchons sera âgée de trente ans ou plus et connaîtra parfaitement les langages Fortran, Snobol, etc. Elle dirigera une équipe composée de 4 opérateurs et opératrices.

- GENS DE MAISON. Employés des deux sexes, capables de fournir excellentes références, pour travail dans grande maison à Westmount. Cuisine, entretien. Permis de conduire, classe "chauffeur».

- ADJOINT(E) AUX OPÉRATIONS D'UNE ENTREPRISE DE TRANSPORT PAR CAMION. Qualifications : connaissance des moteurs diesel, maniement des camions à remorque. Petit capital disponible si possible.

- TECHNICIEN(NE) - ARTS GRAPHIQUES. Homme ou femme pour superviser opérations diverses, département arts graphiques, grande maison en voie d'expansion. Excellentes conditions de travail. Avantages sociaux.

- SOUFFLEUR DE VERRE (HOMME OU FEMME). Si vous avez cinq ans d'expérience dans la fabrication des flacons utilisés en parfumerie nous avons un poste pour vous.

- FACTEURS / FACTRICES. Personnes jeunes et en bonne santé, aimant la vie au grand air, pour assurer la distribution du courrier dans une région rurale éloignée.

IRÈNE V. SPILKA 